\title{
Adenoid cystic carcinoma of the upper airway mimicking a thyroid tumor: A case report
}

\author{
TURKI ALDREES $^{1}$, ABDULLAH ALANAZI ${ }^{2}$, HANADI A. FATANI ${ }^{3}$, \\ ABDULHADI SAMMAN $^{4}$ and SALEH F. ALDHAHRI ${ }^{5}$
}

\begin{abstract}
${ }^{1}$ Department of Otolaryngology-Head and Neck Surgery, College of Medicine, Prince Sattam Bin Abdulaziz University, Riyadh 11942; ${ }^{2}$ Department of Otolaryngology-Head and Neck Surgery, King Faisal Specialist Hospital and Research Centre, Riyadh 12713; ${ }^{3}$ Department of Pathology and Laboratory Medicine, King Fahad Medical City, Riyadh 11525; ${ }^{4}$ Department of Pathology, College of Medicine, University of Jeddah, Jeddah 21589; ${ }^{5}$ Department of Otolaryngology-Head and Neck Surgery, College of Medicine, King Saud University, Riyadh 12372, Saudi Arabia
\end{abstract}

Received February 24, 2016; Accepted May 25, 2016

DOI: $10.3892 / \mathrm{mco} .2016 .931$

\begin{abstract}
Adenoid cystic carcinoma (ACC) is a rare malignant tumor, reportedly representing $<1 \%$ of all head and neck cancers. There have been few reported cases of ACC of the upper airway presenting as a midline mass. This is the case report of a 47-year-old female patient who presented with such a midline neck mass. The mass was approached and investigated as a thyroid mass, but was ultimately found to be a tracheal tumor with thyroid invasion. We herein discuss in detail the patient history, investigation and treatment.
\end{abstract}

\section{Introduction}

Adenoid cystic carcinoma (ACC) was first described by Spies in 1930 (1). It constitutes a rare, malignant tumor, reportedly representing $<1 \%$ of all head and neck cancers. ACC may arise at various sites in the body, including the salivary glands. However, the minor salivary glands of the oral cavity are the most common site, comprising $60 \%$ of all cases (2).

ACC typically grows slowly, but carries a high overall recurrence rate $(\leq 53 \%)$ after initial primary treatment. The risk of recurrence increases in cases with perineural or vascular invasion, positive resection margins and solid histological characteristics. Moreover, the recurrence rate is generally higher in women (3). Evidence of vascular invasion and positive margins may be used as predictors of tumor recurrence $(2,4,5)$.

As the presence of this tumor at sites other than the head and neck is quite rare, and since the progression of the disease

Correspondence to: Dr Turki Aldrees, Department of Otolaryngology-Head and Neck Surgery, College of Medicine, Prince Sattam Bin Abdulaziz University, King Abdullah Street, Riyadh 11942, Saudi Arabia

E-mail: pt.turki@hotmail.com

Key words: adenoid cystic carcinoma, thyroid, tumor, mimicking is occasionally indolent, accurateand timely diagnosis may be difficult.

In this report, we present a rare case of upper tracheal/subglottic ACC mimicking thyroid carcinoma at presentation and the detailed diagnostic process that led to treatment.

\section{Case report}

A 47-year-old woman with known hypothyroidism presented with a history of gradual progressive neck swelling for 6 months, a cough persisting for 4 months, shortness of breath and hoarseness for 1 week. The patient was referred to the Department of Otolaryngology at King Fahad Medical City (Riyadh, Saudi Arabia). As part of the diagnostic investigations, the patient underwent flexible bronchoscopy that revealed no abnormal findings. The pulmonologist at King Fahad Medical City diagnosed the patient with asthma, and treated the respiratory symptoms with bronchodilators and inhaled steroids. The patient experienced some improvement, but her symptoms were not completely eliminated. The patient had no history of weight loss, dysphagia, pain, or exposure to radiation, and no family history of malignancy. In addition to antiasthmatic inhalers, the patient was prescribed levothyroxine (50 $\mu \mathrm{g}$ daily) and antihypertensive medications (amlodipine, $10 \mathrm{mg}$ ).

Physical examination revealed a firm midline mass with smooth, regular borders, sized $\sim 3 \times 4 \mathrm{~cm}$, with no palpable cervical lymph nodes. The mass was located right below the level of the cricoid cartilage and moved with swallowing. Flexible laryngoscope examination revealed left vocal cord paralysis. Routine laboratory investigations, including thyroid function tests, were within the normal limits. An ultrasound revealed a normal-sized right thyroid lobe, with a left lobe measuring $1.7 \times 1.6 \times 4.4 \mathrm{~cm}$. Although both lobes exhibited heterogeneous echotexture, it was more prominent in the left lobe, which demonstrated increased vascularity with no discrete nodules. Fine-needle aspiration (FNA) of the thyroid reported findings consistent with follicular neoplasm/suspicious for follicular neoplasm. Computed tomography (CT) revealed diffuse enlargement of the thyroid 


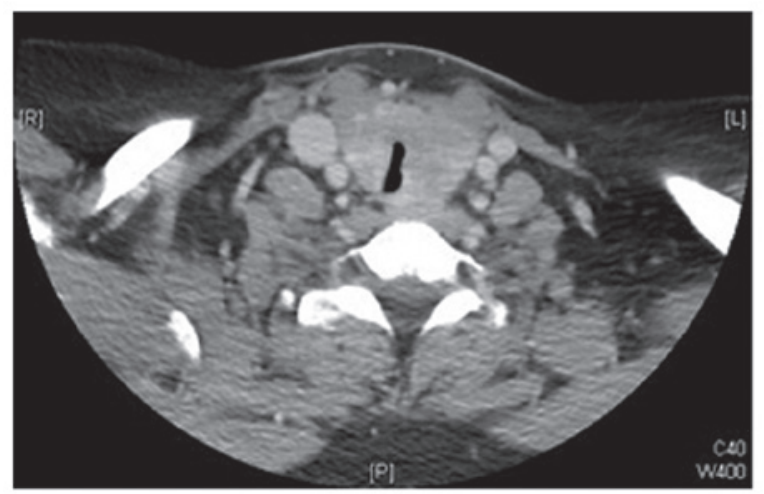

Figure 1. Preoperative computed tomography scan showing significant tracheal compression.

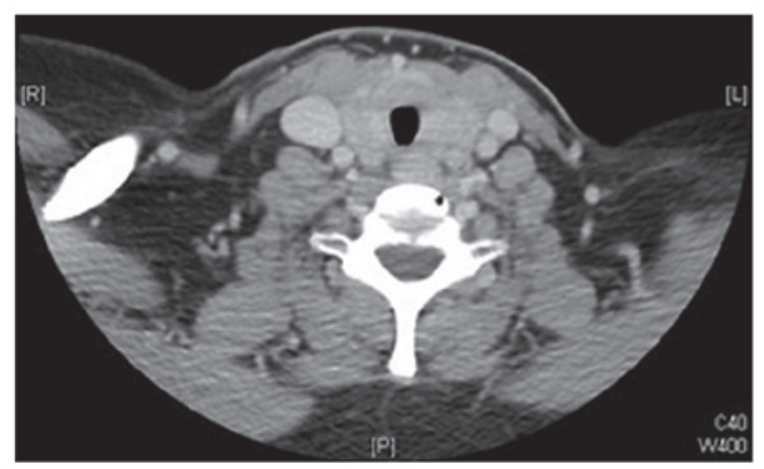

Figure 2. Preoperative computed tomography scan showing diffuse thyroid enlargement.

gland, more prominent on the left side, with significant tracheal compression and possible underlying infiltrative processes, but no cervical lymphadenopathy (Figs. 1 and 2). Based on the FNA and imaging results, the patient was scheduled to undergo total thyroidectomy and possible tracheal resection. Intraoperatively, the thyroid lobes were small and hard, with no gross invasion of the recurrent laryngeal nerve, apparent gross invasion of the trachea, and surrounding edema. As the intraoperative findings were atypical of thyroid carcinoma, frozen specimen sections from the tracheal wall, thyroid gland and left recurrent laryngeal nerve were analyzed. The results reported poorly differentiated carcinoma with adenoid cystic features in the tracheal wall and thyroid gland. The left recurrent laryngeal nerve was reported negative for malignancy.

The surgeons decided to close and plan a second-stage operation after receiving the final surgical pathology report and discussing the results with the patient. The final pathology report confirmed the diagnosis of ACC with predominant tubular and cribriform patterns, with a positive tracheal resection margin and evidence of perineural invasion. The abdominal and chest CT scans, as well as a positron emission tomography scan, were negative. The patient was subsequently rescheduled to undergo total laryngectomy with bilateral neck dissection and tracheal resection. Intraoperatively, the larynx, left side of the cricoid cartilage and the first two tracheal rings were found to be infiltrated by ACC. Total laryngectomy with partial pharyngectomy and tracheal resection with bilateral neck dissection were completed without complications. The final pathology confirmed the diagnosis of ACC involving the larynx and trachea. The epicenter of the tumor was located in the subglottic region and upper trachea, with extensive submucosal tumor formation involving the entire left hemilarynx. However, all regional lymph nodes were negative for malignancy. The final diagnosis was locally advanced ACC of the trachea, stage T4NOMO.

\section{Discussion}

Rare tumors are difficult to diagnose, particularly when their location is uncommon. Tracheal tumors are classified into three categories, namely benign, primary malignant and secondary malignant. Primary malignant tumors most frequently originate from the epithelium or glandular tissues of the trachea. It is extremely rare for these tumors to arise from the connective tissue of the trachea. The two most common types of epithelial tumors are squamous cell carcinoma and adenoid cystic carcinoma, which occur in generally equal proportions. Squamous cell carcinoma is more common in men, while ACC does not show any gender predilection. Pathologically, squamous cell carcinoma is believed to arise from the tall columnar pseudostratified epithelium of the trachea, whereas ACC arises from the mixed seromucinous glands within the trachea (6).

Patients with ACC may present with a slowly progressive shortness of breath and cough, often misdiagnosed as asthma due to the slow rate of ACC progression. In advanced cases, hemoptysis or stridor may be the main diagnostic characteristics. Some patients may also present with hoarseness and weight loss (7). Although rarely, patients may also present with midline neck swelling $(8,9)$. Due to its non-specific symptoms and slow progression, the mean interval between symptom presentation and correct diagnosis of $\mathrm{ACC}$ has been reported to be 12 months (10). Azar et al reported 6 cases of tracheal ACC; dyspnea and non-productive cough were the most frequent complaints, and the duration of symptoms prior to final diagnosis ranged from 6 to 24 months. Although our patient presented with classic, non-specific clinical findings, a follicular pattern in the FNA led to the initial diagnosis of malignant thyroid neoplasm (11).

The clinical and pathological characteristics of tracheal ACC were first reported by Billroth in $1859(7,12)$. Despite their rarity, these tumors are the most common type of salivary gland neoplasms in the lungs (13). ACC commonly occurs in the trachea or main stem bronchi. ACC characteristics may be found anywhere along the tracheal structure, although they tend to be concentrated in the upper region. Histologically, salivary gland ACC is identical to ACC originating elsewhere, with both often demonstrating a tendency towards submucosal extension.

On microscopic examination, cytological smears consisted of small cells with hyperchromatic nuclei and eosinophilic cytoplasm. However, relying on these characteristics as the only diagnostic criterion may lead to a misdiagnosis, particularly when ACC is encountered outside the salivary glands, as was the case with our patient (9). The FNA in our patient revealed alterations in the follicular cell architecture characterized by cell crowding, microfollicles and dispersed isolated cells, with some atypical cells containing a scant amount of colloid. These characteristics supported the diagnosis of follicular thyroid 
neoplasm. However, this misdiagnosis may be attributed to the fact that the FNA specimen was collected from the part of the tumor invading the thyroid gland. Immunohistochemical staining for thyroglobulin or calcitonin may help confirm the diagnosis of thyroid neoplasms; however, these procedures are not routinely performed without clinical suspicion of other possible diagnoses. There are three recognized histological subtypes, namely tubular, cribriform and solid, with the cribriform type being the most common.

One classic feature of ACC is frequent perineural invasion (14), accounting for an increased likelihood of positive microscopic surgical margins at tracheal resection. Regional nodal metastasis has been reported to be $\sim 10 \%$. Over $50 \%$ of patients with tracheal ACC develop hematogenous spread, commonly to the lungs (11). In the present case, however, the patient's chest, abdomen and pelvic preoperative CT scans revealed no distant metastases.

Surgical resection is the treatment of choice for tracheal tumors, whether benign or malignant. The type of surgery is determined based on the location and extent of the tumor. The standard surgery for ACC is sleeve resection of the trachea with primary tracheal anastomosis (15). When ACC is located in the upper trachea, tracheal resection combined with total laryngectomy or laryngopharyngectomy may be necessary, after which a permanent tracheal stoma is required. When ACC involves the carina and main bronchi, a variety of carinal resections and reconstructions are required, involving more intricate surgical techniques. Cricotracheal resection may also be performed in the presence of a subglottic tumor extending to the proximal trachea. Surgical complications usually relate to the extent of the surgery and the clinical status of the patient, and may include anastomotic or wound dehiscence, tracheoesophageal fistula, pharyngeal or esophageal leaks, vocal cord palsy, or dysphagia due to pharyngeal stricture. However, such complications and the resultant mortality have decreased significantly in last decade (16).

Radiotherapy is usually recommended for incomplete resections or positive margins, as it may provide additional local disease control $(7,14)$. The recommended dose is 54-60 Gy (17). Silverman et al (18) performed a retrospective study to determine the benefits of postoperative radiation therapy in patients with ACC of the head and neck. Their results indicated that the addition of postoperative radiation therapy may improve overall survival in patients with advanced T-stage tumors and improve locoregional control in patients with microscopically positive margins. However, the therapy showed no demonstrable benefit in patients with negative margins.

As our patient was diagnosed with an advanced T-stage tumor (T4), she received a radical dose of radiotherapy of 64 Gy using the intensity-modulated radiation therapy technique (18).

Combined-modality therapy with surgery and postoperative radiation may improve locoregional control, particularly in advanced-stage disease. Published reports on locoregional control rates for ACC treated with surgery alone range from 40 to $46 \%$ at 5 years and from 21 to $25 \%$ at 10 years, compared with the locoregional control rates for ACC treated with surgery and postoperative radiation (64-95\% and 68-83\%, respectively). Patients with stage III or IV tumors are most likely to benefit from combined-modality therapy, with locoregional control rates of $16.8 \%$ with surgery alone vs. $51.3 \%$ with surgery and radiation (18). The majority of clinicians hypothesize that the additional benefit of postoperative radiation therapy of ACC of the head and neck would translate into the same benefit when used in the treatment of tracheal ACC. However, no randomized control studies have shown any additional benefit with adjuvant radiotherapy.

The overall survival rate of tracheal ACC has been reported to range between $65-79 \%$ at 5 years and $53-57 \%$ at 10 years (19). Gessrted et al reported a 5-year survival rate of $52.4 \%$ for resected ACC and 33.3\% for unresectable ACC (16). Oplatek et al reported that patients with advanced-stage tumors had a lower median 10-year survival rate compared with patients with stage I or II tumors. The median survival rate was 68 and 61 months for stage III and IV disease, respectively (20).

It remains unclear whether treatment of tracheal ACC with chemotherapy, either as adjuvant or mainstay treatment, carries any clear benefit (7).

Our patient had a T4-stage tumor with perineural invasion and a high risk of recurrence requiring close follow-up. Accordingly, she was followed up for $>1$ year, without any evidence of recurrence.

In conclusion, the case presented herein should alert clinicians to the rare presentation of thyroid masses that do not primarily originate from the thyroid gland. Thorough evaluation of such masses may result in early diagnosis and timely, more effective treatment.

\section{Acknowledgements}

The present study was supported by the College of Medicine Research Center, Deanship of Scientific Research, King Saud University, Kingdom of Saudi Arabia.

\section{References}

1. Spies JW: Adenoid cystic carcinoma: Generalized metastases in three cases of basal cell type. Arch Surg 21: 365-404, 1930.

2. Zhang CY, Xia RH, Han J, Wang BS, Tian WD, Zhong LP, Tian Z, Wang LZ, Hu YH and Li J: Adenoid cystic carcinoma of the head and neck: Clinicopathologic analysis of 218 cases in a Chinese population. Oral Surg Oral Med Oral Pathol Oral Radiol 115: 368-375, 2013.

3. Dantas AN, Morais EF, Macedo RA, Tinôco JM and Morais Mde L: Clinicopathological characteristics and perineural invasion in adenoid cystic carcinoma: A systematic review. Braz J Otorhinolaryngol 81: 329-335, 2015.

4. Honings J, Gaissert HA, Weinberg AC, Mark EJ, Wright CD, Wain JC and Mathisen DJ: Prognostic value of pathologic characteristics and resection margins in tracheal adenoid cystic carcinoma. Eur J CardiothoracSurg 37: 1438-1444, 2010.

5. Haddad A, Enepekides DJ, Manolidis S and Black M: Adenoid cystic carcinoma of the head and neck: A clinicopathologic study of 37 cases. J Otolaryngol 24: 201-205, 1995.

6. Macchiarini P: Primary tracheal tumours. Lancet Oncol 7: 83-91, 2006.

7. Yang PY, Liu MS, Chen CH, Lin CM and Tsao TC: Adenoid cystic carcinoma of the trachea: A report of seven cases and literature review. Chang Gung Med J 28: 357-363, 2005.

8. Kattepur AK, Patil D, Srinivas KG, Swamy S, Murthy V, Amarendra S and Gopinath KS: Adenoid cystic carcinoma of trachea masquerading as papillary thyroid cancer - a case report. Case Rep Clin Pathol 1: p53, 2014.

9. Idowu MO, Reiter ER and Powers CN: Adenoid cystic carcinoma: A pitfall in aspiration cytology of the thyroid. Am J Clin Pathol 121: 551-556, 2004. 
10. Bagheri R, Fatahi MS, Mokhtari AM and Rahim MB: Adenoid cystic carcinoma of the trachea. Tanaffos 7: 49-54, 2008.

11. Azar T, Abdul Karim FW and Tucker HM: Adenoid cystic carcinoma of the trachea. Laryngoscope 108: 1297-1300, 1998.

12. Khan AJ, DiGiovanna MP, Ross DA, Sasaki CT, Carter D, Son YH and Haffty BG: Adenoid cystic carcinoma: A retrospective clinical review. Int J Cancer 96: 149-158, 2001.

13. Vigg A, Mantri S, Vigg A and Vigg A: Adenoid cystic carcinoma of trachea. Indian J Chest Dis Allied Sci 46: 287-290, 2004.

14. Bonner Millar LP, Stripp D, Cooper JD, Both S, James P and Rengan R: Definitive radiotherapy for unresected adenoid cystic carcinoma of the trachea. Chest 141: 1323-1326, 2012.

15. Suzuki T: What is the best management strategy for adenoid cystic carcinoma of the trachea? Ann Thorac Cardiovasc Surg 17: 535-538, 2011.

16. Gaissert HA, Grillo HC, Shadmehr MB, Wright CD, Gokhale M, Wain JC and Mathisen DJ: Long-term survival after resection of primary adenoid cystic and squamous cell carcinoma of the trachea and carina. Ann Thorac Surg 78: 1889-1896; discussion 1896-1897, 2004.
17. Lay G, Amichetti M, Dessi M, Orru S and Versace R: Adenoid cystic carcinoma (ACC) of the tracheo-bronchial tree treated with laser therapy and irradiation: Report of two cases. The Open Lung Cancer Journal 2: 31-34, 2009.

18. Silverman DA, Carlson TP, Khuntia D, Bergstrom RT, Saxton J and Esclamado RM: Role for postoperative radiation therapy in adenoid cystic carcinoma of the head and neck. Laryngoscope 114: 1194-1199, 2004.

19. Maziak DE, Todd TR, Keshavjee SH, Winton TL, Van Nostrand P and Pearson FG: Adenoid cystic carcinoma of the airway: Thirty-two-year experience. J Thorac Cardiovasc Surg 112: 1522-1532, 1996.

20. Oplatek A, Ozer E, Agrawal A, Bapna S and Schuller DE: Patterns of recurrence and survival of head and neck adenoid cystic carcinoma after definitive resection. Laryngoscope 120: 65-70, 2010. 